\title{
Concurrent three dimensional neuroendoscopy: initial descriptions of application to clinical practice
}

\section{Joseph C. T. Chen, M.D., Ph.D., Michael L. Levy, M.D., Ziv Corber, and Murwarid Mura Assifi}

Division of Neurosurgery, Children's Hospital of Los Angeles, and the Department of Neurological Surgery, University of Southern California School of Medicine, Los Angeles, California

Applications of endoscopic technique neurosurgery are becoming increasingly popular as greater evidence of the safety and efficacy of these techniques is reported. Nevertheless, significant technical limitations need to be solved before neuroendoscopy can achieve widespread popularity. One limitation is the surgeon's difficulties in becoming anatomically oriented in a two-dimensional (2-D) environment. The lack of appropriate visual cues to orient oneself in three-dimensional (3-D) space makes relatively simple anatomical regions difficult to navigate. The authors describe an endoscopy system that allows for stereoscopic visualization during minimally invasive procedures and that acts as an adjunct to conventional open craniotomies. Four cases are described in which stereoendoscopy was used as either a primary means of visualization or as an adjunct to the operating microscope in conventional open neurosurgical procedures. The authors believe that stereoendoscopic vision is a significant advance in endoscope technology and will play a large role in the popularization of minimally invasive techniques in neurosurgery.

\section{Key Words * endoscopy * neuroendoscopy * head-mounted display * computerized imaging}

Previous goals in refining endoscopic surgical technique have been reductions in cost, reduced rates of morbidity, and increased patient satisfaction. These factors have been the principal driving forces for the introduction of endoscopic procedures into nearly every branch of the surgical disciplines. Furthermore, a more informed patient base has begun to demand surgical procedures that are associated with minimum cosmetic damage and more favorable side-effect profiles. Despite the potential advantages of endoscopy, the techniques have been associated with a steep learning curve, largely caused by the relatively low-quality of visual information conveyed by endoscopy in contrast to the operating microscope. Rather than providing a direct stereoscopic view of the operative field, endoscopic visual information is most commonly conveyed as 2-D images projected onto a video screen. Previously, we conducted evaluations of improvements in display technology to ergonomically improve the effectiveness of endoscopic surgical procedures. In this report, we examine the use of a 3-D or stereoendoscopy system. This 
communication complements our previous reports on stereoscopic head-mounted display technology.[3, 11]

\section{CLINICAL MATERIALS AND METHODS}

Stereoendoscopic methods were used as adjunctive measures to open microneurosurgical procedures, performed under the guidelines of the Institutional Review Board, conducted at the Children's Hospital of Los Angeles in 1998.

\section{Description of Equipment}

A neurostereoendoscope, fabricated to our specifications by Vista Medical Technologies (Carlsbad, CA), was used (Fig. 1). This endoscope has an external diameter of $4.7 \mathrm{~mm}$ and uses a single glass rod optical element. A prismatic optical path separator is mounted behind the rod lens, and dual charged-coupled devices (CCDs) of $640 \times 480$ matrix were used to capture stereoscopic images. The effective interpupillary distance is $1.0 \mathrm{~mm}$ with a working distance of $15 \mathrm{~mm}$. Video images were processed on a processor (Vista StereoSite, Vista Medical Technologies, Carlsbad, CA) to convey images to a head-mounted display.[11] Stereoscopic video images were archived on a wide VHS digital video recorder for later retrieval and hard-copy image production.

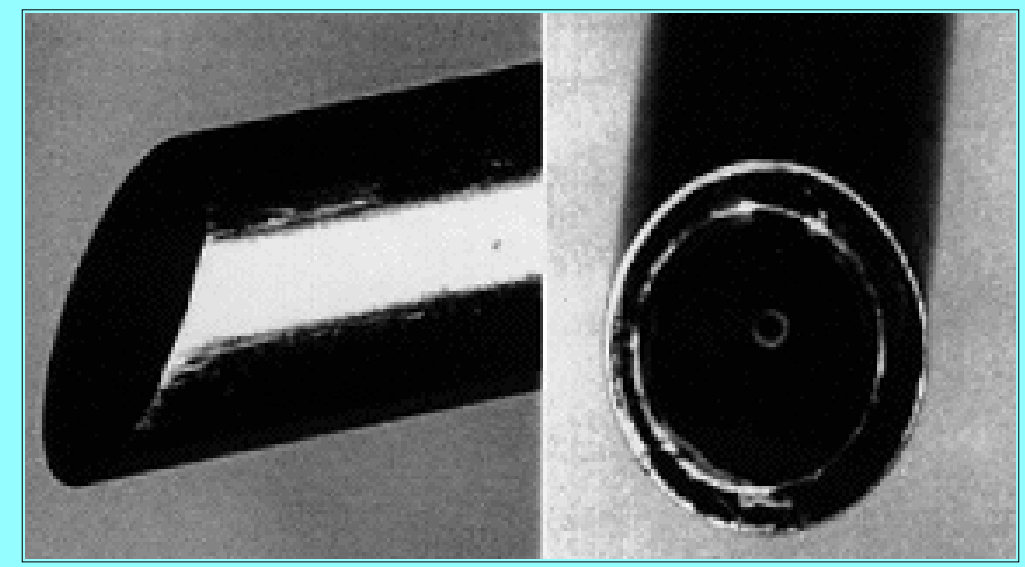

Fig. 1. Photograph displaying neurosurgical endoscope, with tip diameter of $4.7 \mathrm{~mm}$ and $30^{\circ}$ view angle A single glass rod lens is used with a prism image splitter and CCD assembly located in the handpiece.

The current system allows for picture-in-picture capabilities that can be prompted using voice commands. The optics of the system allow for the concurrent visualization of the operative field (3-D), endoscopic field (3-D), 3-D virtual reconstructions of the subarachnoid space or ventricular chamber, or frameless stereotactic information. The system was initially tested in the laboratory setting, as previously reported, before being used in the operating room (OR) (Fig. 2). 


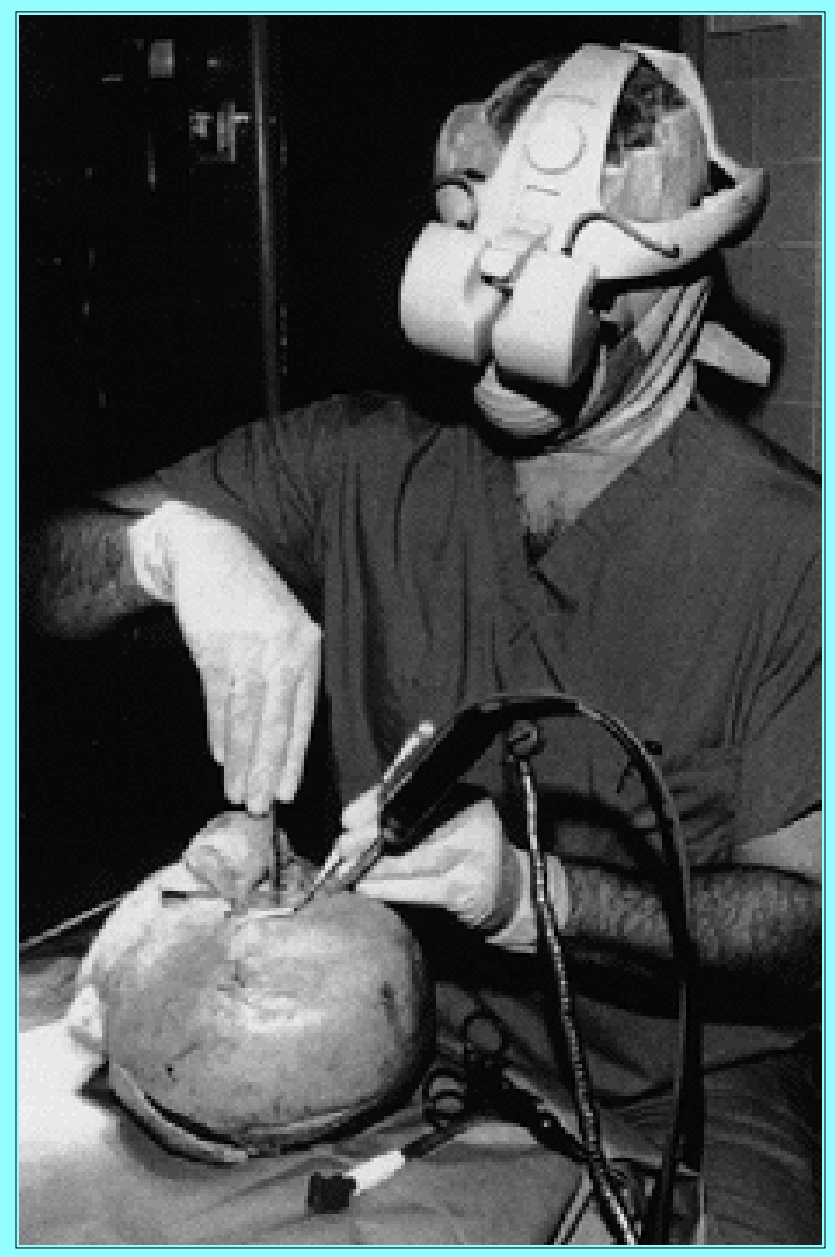

Fig. 2. Use of neurosurgical endoscope in the laboratory setting. Here, images are displayed simultaneously in stereoscopic format in the surgeon's head-mounted display.

\section{RESULTS}

\section{Endoscopic-Assisted Temporal Craniotomy for Fenestration of Arachnoid Cyst}

We have used stereoendoscopy as an adjunct to continually obtained visualization in two cases in which arachnoid cysts were treated. It is the standard practice of our group to perform microsurgical fenestration of arachnoid cysts as the primary treatment, as has been previously indicated.[6] Endoscopic fenestration of arachnoid cysts is also becoming a popular option in the therapy of these lesions.[7,12]

An 18-year-old patient presented to our facility with a history of progressive headaches. We obtained a noncontrast computerized tomography scan that revealed an arachnoid cyst in the right middle fossa (Fig. 3 ), and a right temporal key-hole approach was performed for cyst fenestration. Following microsurgical fenestration of the cyst, evaluation of the fenestration into the basilar cisterns and visualization of the third cranial nerve along the tentorial incisura was completed (Fig. 4). 


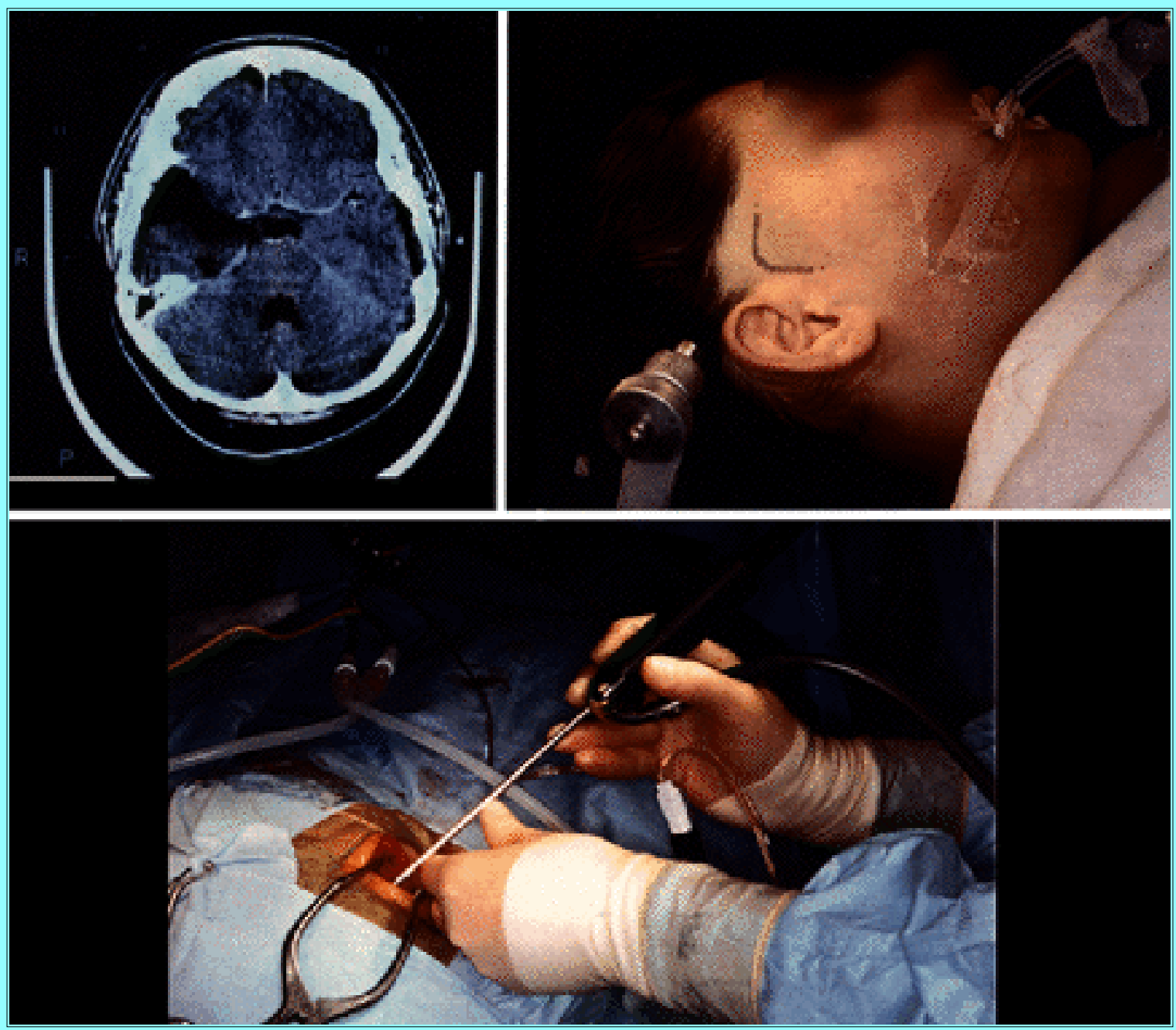

Fig. 3. Fenestration of an arachnoid cyst located in the middle fossa. Upper Left: A computerized tomography scan revealing cyst in the right temporal fossa. Upper Right: An incision is made. Lower: The endoscope is inserted into the key-hole craniotomy.
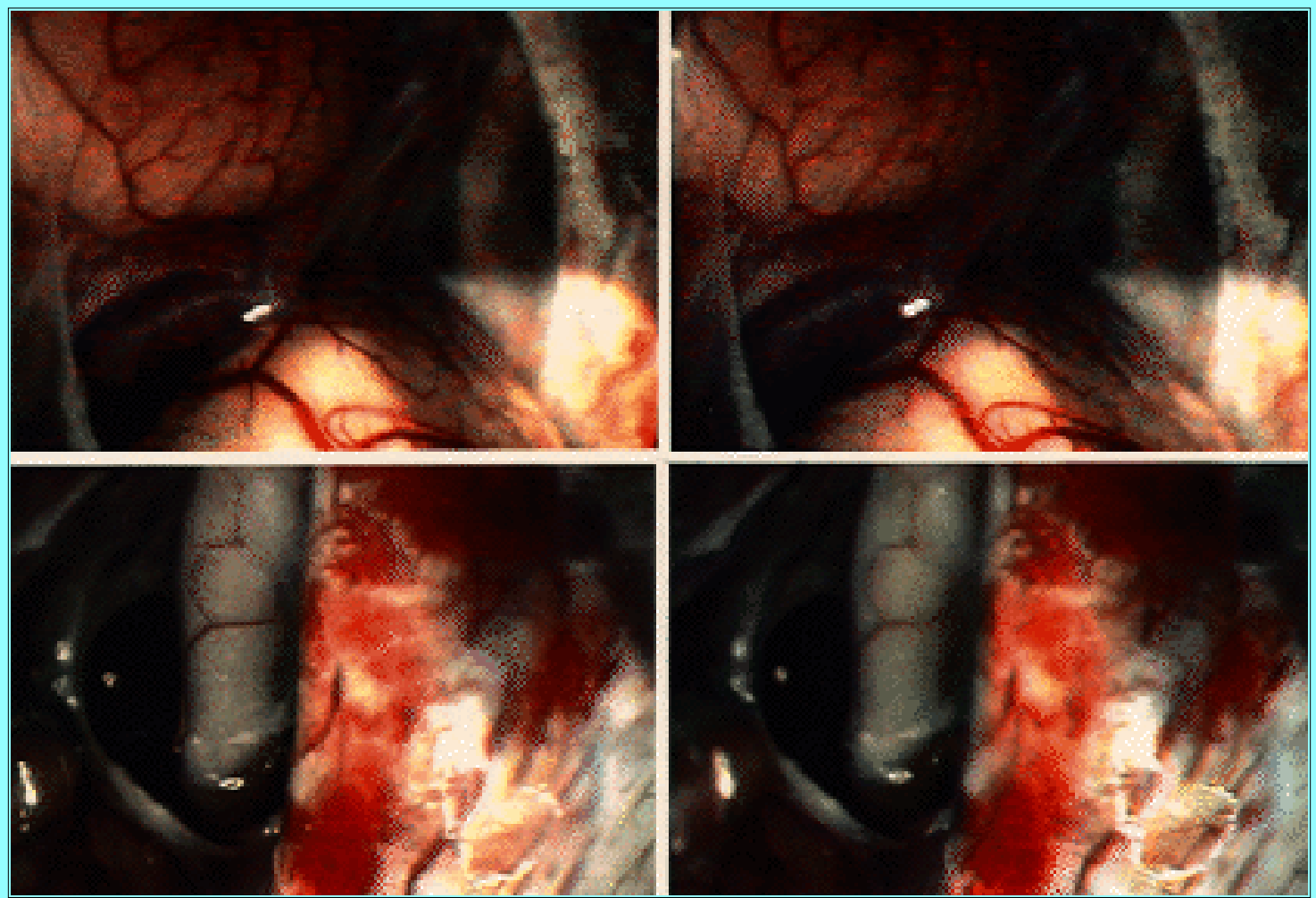
Fig. 4. Stereograms constructed from simultaneously acquired video images from left and right channels of endoscope during fenestration of arachnoid cyst. Left: Initial visualization of medial portion of cyst. Note third cranial nerve and middle cerebral artery branches.

Right: Postfenestration view of medial cyst wall.

\section{Endoscopic Assisted Craniotomy for Removal of Craniopharyngioma}

Three children who harbored craniopharyngiomas with suprasellar extension underwent endoscopic-assisted craniotomy. One of the three children presented with a large craniopharnygioma with suprasellar extension and superior extension into the third and lateral ventricles. A transbasal approach was performed, and resection was aided by endoscopic-assisted visualization through the lamina terminalis. Endoscopy in this case was also used in conjunction with interactive image-guided surgical techniques (Fig. 5).

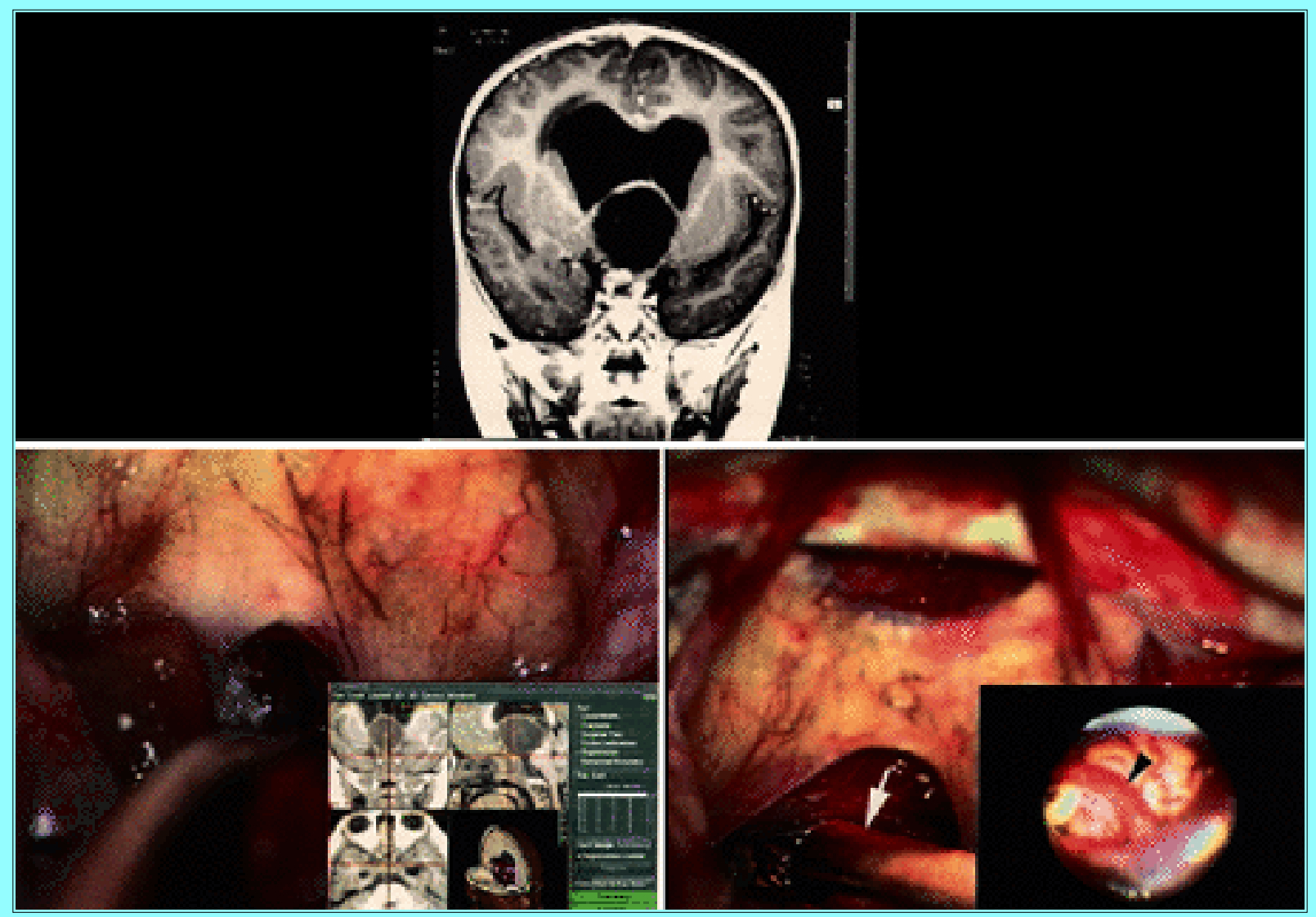

Fig. 5. Various imaging studies demonstrating endoscopic-assisted resection of suprasellar craniopharyngioma. Upper: Magnetic resonance (MR) image demonstrating position of the lesion. Lower Left: Advanced operative display with picture-in-picture image guidance was used in the frameless interactive image-guided surgery. Lower Right: Concurrent microsurgical and neurendoscopic views overlayed in picture-in-picture format. Endoscopic visualization of basilar artery obtained through lamina terminalis. Note clear visualization of origin of superior cerebellar artery from basilar artery (black arrow head) and the trajectory of endoscope (white arrow).

Following initial interhemispheric approaches, endoscopic visualization of the third ventricle was continued concurrently with the temporopolar approach.[6] Concurrent 3-D endoscopic visualization allowed for verification of complete resection of the third ventricular component. After the temporopolar approach, resection of the tumor was endoscopically verified via the translaminar approach. 


\section{Click here to view Video.}

Simultaneous external and through-scope video demonstrating use of scope in craniopharyngioma resection via an orbitozygomatic approach. Note view of basilar artery through lamina terminalis--a view not obtainable by the conventional operating microscope.

The concurrent stereoendoscopic images obtained during this approach yielded visual access to the third ventricle in a manner not possible with the straight visual path of the microscope. A $30^{\circ}$-angled endoscopic image was obtained that yielded excellent endoscopic images.

\section{DISCUSSION}

Endoscopic procedures have had a long history in neurosurgery dating back to the work of Dandy, Mixter, and others. Despite important work conducted by these early investigators, neuroendoscopy fell into disuse, largely because of alternate technologies that made the procedures for which the endoscope was developed obsolete. On a sporadic basis endoscopy has been reintroduced to the neurosurgical armamentarium to be used in the diagnosis and treatment of intraventricular tumors as well as an adjunctive visualization modality for open microsurgical procedures.

One factor accounting for the reacceptance of endoscopy into surgical disciplines has been the improved visualization offered by modern endoscopic devices. Each major advance in endoscopic surgery has been driven by improvements in visualization technology rather than changes in the particular endoscopic surgical instruments. In initial endoscopic visualization a direct view was obtained by the surgeon through the eyepiece of the endoscope. This limited the view of the operative field to the surgeon, whereas others in the OR setting had no view through the endoscope. This limitation certainly made the procedures more difficult, as surgical assistants could do nothing in the operative field. Furthermore, it was difficult to train people in these techniques, because surgeons and trainees could not simultaneously view the operative field.

A revolution occurred in the early 1980s when small CCDs replaced the Vidicon tubes previously used for video applications. The CCD camera was lightweight, durable, and could be mounted to the eyepiece of the endoscope. The video image could then be projected, allowing numerous OR personnel to view the operative field simultaneously. The introduction of this new visualization modality made possible the introduction of more complex minimally invasive surgical techniques.[1,8,14-17]

However, with a few exceptions the neurosurgical field has been slow to adopt new endoscopic methods. The reasons for this are sundry. Among them are inferior visualization compared with that which can be obtained through an operating microscope, as well as the steep learning curve associated with these procedures. This is a particularly important issue in neurosurgical procedures in which narrow operative corridors and long reaches limit the number of visual depth cues available to surgeons performing surgery in these areas. Good visualization, therefore, is essential to facilitate microdissection and prevent damage to nearby sensitive structures.[13]

A critical limitation to the use of endoscopic technology has been the loss of stereoscopic information. Stereoscopic vision is a critical means of depth perception.[10] To the best of our knowledge, this is the first report of the use of stereoendoscopic technology in neurosurgical intracranial procedures. In recent years, a number of groups have used stereoendoscopic methods in other organ systems.(Calano, et al.,[2], 
Simon,[18] and unpublished data). Most notably, in investigations of the gastrointestinal system, stereoendoscopy may allow optical measurements of normal or diseased structures. Similar applications are found in the neurosurgical arena.

A significant obstacle to the introduction of stereoscopic endoscopes has been the end-display technology. In early 3-D endoscopy procedures of the upper airway the endoscope had a 6-mm diameter and $0^{\circ}$ angulation. The endoscope was supplied with a 100-Hz monitor and high-speed liquid crystal shutter glasses. The 3-D image was based on obtaining alternating sequential projections of left and right eye images on a single video monitor. Two high-speed liquid crystal stereo switches were integrated in glasses, and a rapid switching time of 100 microsec was used.[19] Most video stereoscopic displays have been cumbersome, and in the implementation of technological solutions, significant degradation in image quality has resulted. The head-mounted display device provides an excellent ergonomic solution and provides good image quality.

Despite the theoretical advantages of stereoscopic display technology, data regarding task performance by using stereoscopic compared with monoscopic displays are unclear. A number of investigators have undertaken performance studies in which various standardized tasks are used. In general, it appears that stereoscopic vision does not seem to improve speed or the rate of error in the hands of experienced surgeons.[4,9,20] With students or inexperienced surgeons, however, there does appear to be a reproducible, if small, advantage to stereoscopic vision.[4,9,20] Notable, however, is that when tasks are performed using direct vision through the endoscope, significant improvement in performance is demonstrated.[20] The data therefore seem to suggest that a number of other factors in video processing, display, and endoscope optics conspire to degrade the ability to perceive important cues that may facilitate task performance. These factors have not been adequately identified to date.

Although these studies have been conducted to examine the use of stereoscopic displays in standardized tasks, it is unclear how the indices of performance on these tasks can be translated into the operative environment. In difficult neurosurgical procedures, such as the resection of tumors located in regions of potentially difficult anatomy and in those procedures for which there are few stereotyped tasks, use of stereoscopic displays may confer significant advantages to the surgeon and team. In our experience with stereoendoscopic technology, we have found that these devices will likely play an important role in the future development of microneurosurgical techniques. Furthermore, they allow visualization of structures through exposures not otherwise accessible when using the conventional operating microscope.

\section{References}

1. Anonymous: [Introduction of various types of commercially available electronic endoscopes and related equipment.] Nippon Rinsho 45:1313-1323, 1987 (Jpn)

2. Catalano MF, Van Dam J, Bedford R, et al: Preliminary evaluation of the prototype stereoscopic endoscope: precise three-dimensional measurement system. Gastrointest Endosc 39:23-28, 1993

3. Chen JC, Moffitt K, Levy ML: Head-mounted display system for microneurosurgery. Stereotact Funct Neurosurg 68:25-32, 1997

4. Eguchi S, Kohzuka T, Araie M: Stereoscopic ophthalmic microendoscope system. Arch Ophthalmol 115:1336-1338, 1997

5. Fewel ME, Levy ML, McComb JG: Surgical treatment of 95 children with 102 intracranial arachnoid 
cysts. Pediatr Neurosurg 25:165-173, 1996

6. Hakuba A, Liu S, Nishimura S: The orbitozygomatic infratemporal approach: a new surgical technique. Surg Neurol 26:271-276, 1986

7. Hopf NJ, Perneczky A: Endoscopic neurosurgery and endoscope-assisted microneurosurgery for the treatment of intracranial cysts. Neurosurgery 43:1330-1337, 1998

8. Itoshima T, Tanaka R, Ando M, et al: [Image processing devices related to electronic endoscopy.] Nippon Rinsho 45:1174-1179, 1987 (Jpn)

9. Jones DB, Brewer JD, Soper NJ: Next-generation 3-D video systems may improve laparoscopic task performance, in Satava RM (ed): Interactive Technology and the New Paradigm for Healthcare. Washington, DC: IOS Press, 1995, pp 190-212

10. Julesz B: Stereoscopic vision. Vision Res 26:1601-1612, 1986

11. Levy ML, Chen JC, Moffitt K, et al: Stereoscopic head-mounted display incorporated into microsurgical procedures: technical note. Neurosurgery 43:392-396, 1998

12. McGregor JM: Enhancing neurosurgical endoscopy with the use of virtual reality' headgear. Minim Invasive Neurosurg 40:47-49, 1997

13. Mennel H, Rossberg C: "Stereology" of intracranial lesions. Acta Neurochir Suppl 61:62-68, 1994 14. Mitchell T, Robertson J, Nagy AG, et al: Three-dimensional endoscopic imaging for minimal access surgery. J R Coll Surg Edinb 38:285-292, 1993

15. Morikubo H, Muto K, Ikeguchi S, et al: [Comparison of various types of commercially available electronic endoscopes based on personal experiences.] Nippon Rinsho 45:1306-1312, 1987 (Jpn)

16. Rasinger R, Neuwirth-Riedl K, Gajsek M, et al: [Clinical experiences with digital video image analysis procedures in the assessment of endoscopic ENT video findings.] Wien Klin Wochenschr 00:20-23, 1988 (Ger)

17. Schwing C: [Endoscopy in transition. Video endoscopes replace glass fiber systems--uses of the new technic?] Fortschr Med 106:56-57, 1988 (Ger)

18. Simon W: A simple stereoscopic endoscope. J Soc Laparoendosc Surg 2:67-70, 1998

19. Stultz J: Die 3-D endoscopy. HNO 41:128-130, 1993

20. Tendick F, Bhoyrul S, Way LW: Comparison of laparoscopic image systems and conditions using a knot-tying task. Comput Aid Surg 2:24-33, 1997

Manuscript received February 24, 1999.

Accepted in final form March 11, 1999.

Address reprint requests to: Michael L. Levy, M.D., 1300 North Vermont Avenue, Suite 906, Los Angeles, California 90027. 


\section{QuickTime Movies}
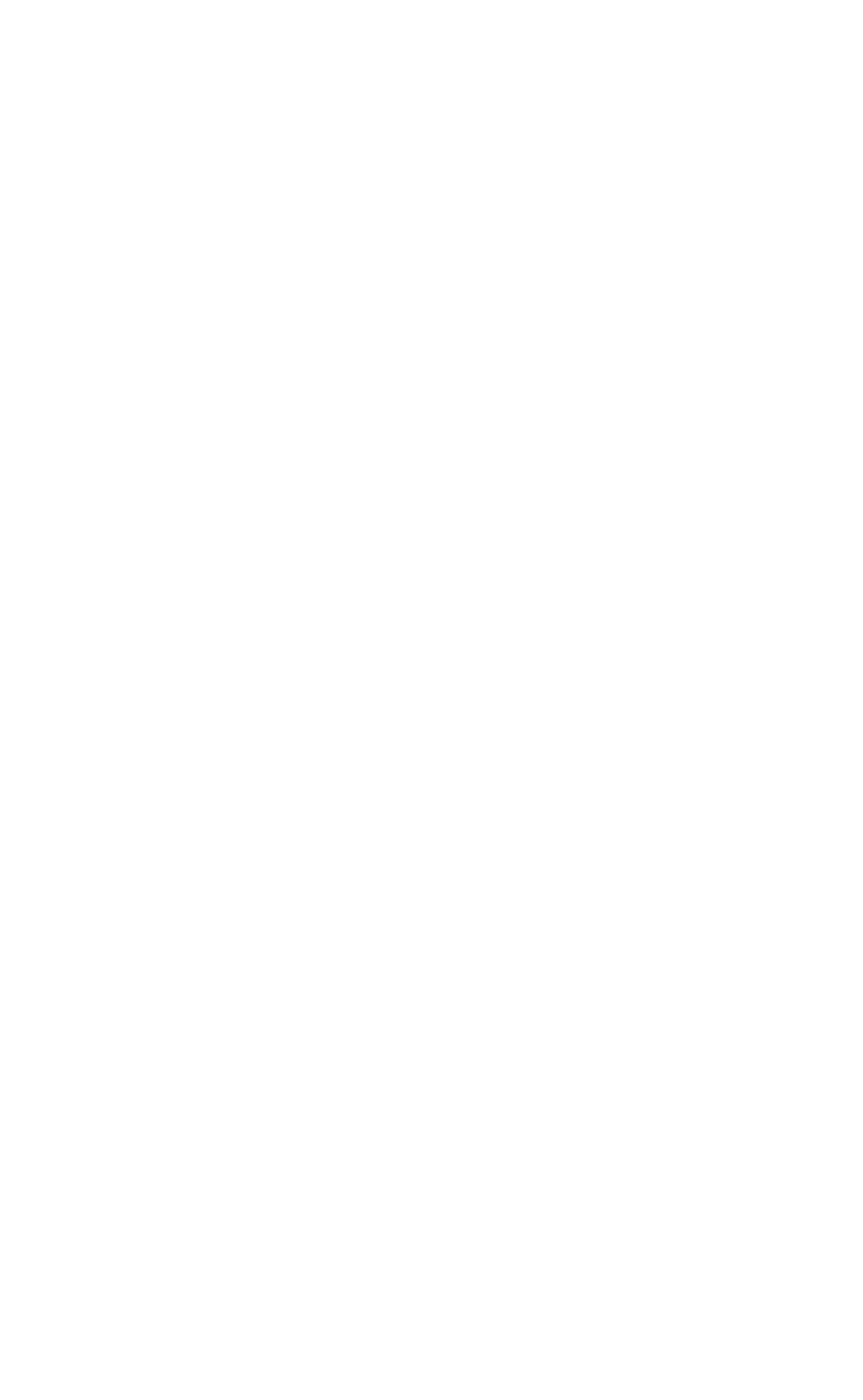